\title{
Analysis of landscape structure, habitat selection and urbanisation in edge populations of Scops Owls Otus scops in Central Europe ${ }^{x}$
}

\author{
Ákos KLeIN ${ }^{1 *}$, István SzentiRmaI ${ }^{2}$, Zsófia Dobos ${ }^{1} \&$ Miklós LaCZI $^{1,3}$
}

${ }^{\times}$Presented at $2^{\text {nd }}$ Hungarian Owl Research Conference held in Pécs on $11^{\text {th }}$ September 2020.

Received: October 14, 2020 - October 23, 2020 - Accepted: October 30, 2020

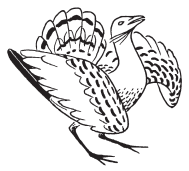

Klein, Á., Szentirmai, I., Dobos, Zs. \& Laczi, M. 2020. Analysis of landscape structure, habitat selection and urbanisation in edge populations of Scops Owls Otus scops in Central Europe.Ornis Hungarica 28(2): 24-36. DOI: 10.2478/orhu-2020-0015

\begin{abstract}
The habitat selection of Scops Owl Otus scops has not been studied in Hungary so far. The population in the Carpathian Basin can be considered as a range edge population. Yet, studying and conserving breeding population at the edge of the species' range is important for the evolutionary potential of the species. In the present study, we examined Scops Owl populations situated on both sides of the Hungarian-Slovenian border. Although breeding density is significantly higher in Slovenia than in Hungary, we found no difference in the ecological diversity of the Goričko Nature Park (GNP), Slovenia and Vas County, Hungary. We found that both the proportion and total edge length of dry grasslands and intensively managed mesic grasslands were lower in Hungary. Similarly, market gardens were present in a larger proportion in GNP. These landscape features all indicate that the complex cultivation is still pronounced in GNP, favouring the Sops Owl as less intensive cultivation modes, like rural market gardens and grasslands play a key role in its habitat selection. Points with Scops Owl observations appeared to be closer to settlements than randomly generated points. They also were observed farther from primary roads than from secondary roads. This is in accordance with other studies revealing that these nocturnal birds avoid noisy roads. We briefly discuss why conserving range edge populations is important, and how time and effort optimised species conservation measures should accompany landscape protection at the political level.
\end{abstract}

Keywords: range edge population, farmland bird, common agriculture policy, predation, Tawny Owl

Összefoglalás A füleskuvik Otus scops a Kárpát-medencében perempopulációnak tekinthető az európai állomány zömét alkotó mediterrán népességhez képest. Mégis, az elterjedési terület szélén elhelyezkedő költőállomány vizsgálata és védelme evolúciós szempontból fontos. A jelen tanulmányban a magyar-szlovén határ két oldalán megtalálható, denzitásában jelentősen eltérő füleskuvik állományt vizsgáltuk. Nem találtunk eltérést a Goričko Tájpark és Vas megye ökológiai diverzitásában. Kimutatható volt, hogy a szlovén mintaterületen magasabb arányban vannak jelen a komplex müvelésre utaló mezőgazdasági kultúrák (száraz gyepek, közepesen intenzíven kezelt gyepek, település közeli zöldségeskertek). E müvelési ágak egymás melletti sokfélesége kedvez a füleskuvikok megtelepedésének. Kimutattuk, hogy a füleskuvikok a véletlenszerühöz képest közelebb helyezkednek el a településekhez és a településekhez kötődő utakhoz, de az elsőrendü, forgalmasabb úttípusoktól távolabb fordulnak elő. Eredményeink erősítik a korábban már leírtakat, miszerint az utak elsősorban zajterhelésükkel taszítják a gyakran vokalizáló madarakat. A hazai viszonyokra javasoljuk a tájban kialakítani a veterán fák és állandósult cserjesorok hálózatát, illetve hosszútávon elkerülhetetlen a tájszerkezet további leromlásának megfékezése erőteljes szakpolitikai döntések segítségével.

Kulcsszavak: perempopuláció, Közös Agrárpolitika, urbanizáció, zajterhelés, predáció, macskabagoly 
${ }^{1}$ The Barn Owl Foundation, 8744 Orosztony Temesvári utca 8.

${ }^{2}$ Örség National Park Directorate, 9941 Öriszentpéter, Városszer 57.

${ }^{3}$ Behavioural Ecology Group, Department of Systematic Zoology and Ecology, Eötvös Loránd University,

1117 Budapest Pázmány Péter sétány 1/C

*corresponding author, e-mail: barnowlfoundation@gmail.com

\section{Introduction}

The Scops Owl Otus scops is the only long-distance migrant European owl species. Its largest population can be found in the Mediterranean. Its spatial distribution in Europe is constrained by the chains of Alps and Carpathians, and this species reaches north in Asia as far as the $16{ }^{\circ} \mathrm{C}$ isotherm line (Gill \& Donsker 2019). The total European population is cca. 232,000-393,000 pairs that constitutes approximately $60 \%$ of the world population (BirdLife International 2020).

Both the large geographic range and population size justify that its IUCN (International Union for Conservation of Nature) status is „least concern”. Contrastingly, it is regarded as one of the most rapidly declining owl species in Europe (Sergio et al. 2009). Several recent publications intended to reveal the most important factors that can explain this decrease (Denac et al. 2019, Ivajnšič et al. 2020).

In many species, populations close to the edge of their range are especially important for their preservation as breeding individuals may often occupy suboptimal habitats, hence evolutionary forces can act differently to the central populations. Highly variable recruitment, lower fecundity, increased population fragmentation and increased adult dispersal are only some of the factors that can contribute to the vulnerability of edge populations (Gaston 2009).

Hungary, Slovakia and Ukraine constitute the Northern brim of Scops Owl's European range. A moderate expansion of this species was detectable in the 1950s in the Carpathian Basin, similarly to Syrian Woodpeckers (Dendrocopos syriacus) and Eurasian Collared Doves (Streptopelia decaocto) (Randik 1959). Ornithologists, almost seven decades ago, had already explained this expansion with the increase of the average temperature in Europe (Barthos 1957, Randik 1959).

The distribution of the Scops Owl in Hungary is not exhaustedly mapped yet. The breeding population in Hungary is about 300-600 pairs (Hadarics \& Zalai 2008, MME 2020). The highest population density can be found in Central Hungary (Duna-Tisza Interfluvial), whereas in the Western part of Hungary, only very sporadic breeding records are known. At the same time, in Northeast Slovenia (Goričko Region), 210 calling males were recorded in the investigated area covering $442 \mathrm{~km}^{2}$ in 1997 (Štumberger 2000), and even after a severe $70 \%$ population decline, there are still 60-70 breeding pairs in this region (Denac et al. 2019). Northeast Slovenia and West Hungary are adjacent areas in Central Europe, and they share many similarities in terms of landscape characteristics, climate and geography.

In this study, we endeavoured to reveal why geographically similar areas carry Scops Owl populations of very different sizes. We also tried to find explanations about landscape features that may be accountable for Scops Owl distribution in West Hungary (Vas County). In order to answer these questions, we carried out systematic Scops Owl surveys in 2019. 
Landscape structure was compared between the Slovenian and the Hungarian study areas. We investigated the relationship between road network, settlements and water courses and the spatial distribution of the Scops Owl.

\section{Material and methods}

\section{Study area and data collection}

Data were collected in Vas County (Hungary, 3,336 km², 47 $05^{\prime} 13^{\prime \prime} \mathrm{N}, 16^{\circ} 42^{\prime} 17^{\prime \prime} \mathrm{E}$ ) and in Goričko Nature Park (Slovenia, $463.5 \mathrm{~km}^{2}$, mentioned as GNP hereafter, $46^{\circ} 46^{\prime} 32^{\prime \prime} \mathrm{N}$, $16^{\circ} 11^{\prime} 46^{\prime \prime}$ 'E) (Figure 1). A representative Scops Owl survey was carried out in 2019 in 79 out of the total of 632 UTM quadrates $(2.5 \times 2.5 \mathrm{~km}$ each $)$ that covers $14.8 \%$ of the territories of Vas County. The 79 quadrates were systematically selected: $50 \%$ of them within the area of the National Ecological Network (http://web.okir.hu/map), the other half outside of this network. Data arising from 2019 and previously recorded observations from national databases (www.birding.hu 2006.06.30-2019.04.02., www.map.mme.hu 2008.05.032018.10.09., Örség National Park database 2012.03.17-2018.10.18.) were pooled. Prior to 2019, a total of 43 Scops Owl observations were extracted from the database. In 2019, a total of 10 calling males were observed from the 79 UTM quadrates. Data from Goričko were kindly provided by K. Denac (DOPPS, BirdLife Slovenia). For the analyses, we used a total of 12 Hungarian and 18 Slovenian independent observations. The rest of the data points were multiple observations from the same locations that were excluded from the analyses.

\section{Environmental variables}

The home range of the Scops Owl was defined as a 30 ha circular plot (309 m radius) around the nest site (Martínez et al. 2007). We investigated environmental (habitat) variables within a larger, $500 \mathrm{~m}$ radius circle drawn around the observation points. A land cover map with a resolution of $10 \mathrm{~m} /$ pixel was used to analyse ecological diversity (map created by University of Vienna, M. Pöchtrager in 2019, unpublished). This map was created with the interpretation of Sentinel-2 satellite images (Copernicus Sentinel data 2017 \& 2018) in which each pixel was assigned to one of the EUNIS level-2 habitat types (Davies et al. 2004). 12 out of the total of 22 habitat types were used for the analyses, after we excluded those that were only represented in small proportion of the $500 \mathrm{~m}$ large sample circles (Table 1).

\section{Data and GIS analysis}

We performed Mann-Whitney U-tests on the individual habitat variables to compare the Hungarian and the Slovenian observation points of Scops Owls. We adjusted the significance levels by Benjamini-Hochberg FDR correction (Benjamini \& Hochberg 1995). Then, we explored whether there was any difference between the countries if we treated the habitat variables as composite indices. In order to do this, we performed principal component 


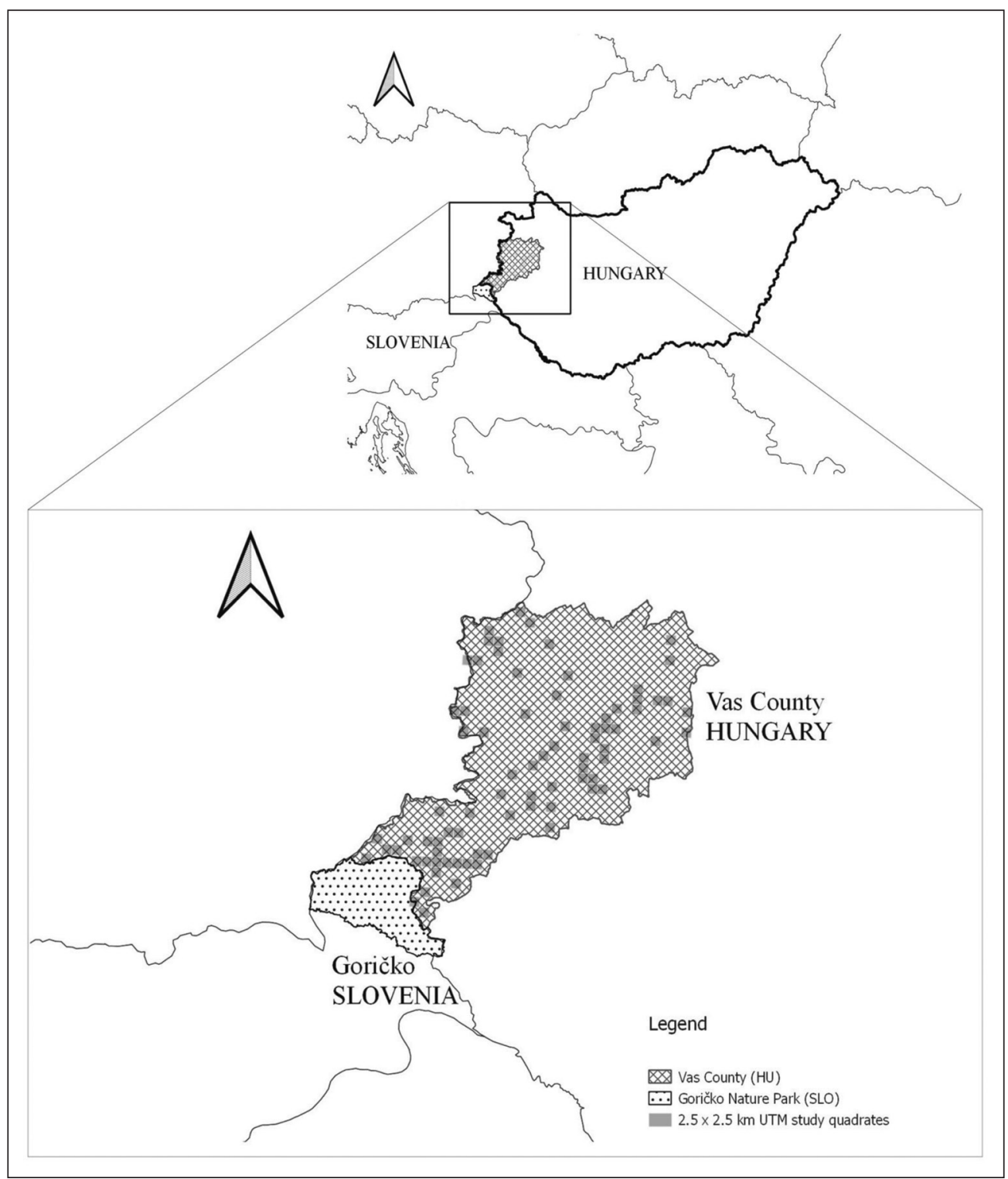

Figure 1. Map of the study area in Hungary (Vas County and in Slovenia (Goričko Nature Park i...)

1. ábra A vizsgálati terület áttekintő térképe (Vas megye Goričko Tájpark

analysis (PCA) on these variables with varimax rotation, and then we compared the principal components (PCs) between countries using Mann-Whitney U-tests. We used in the analyses only PCs of which eigenvalues was larger than one.

In addition, we also applied a Geographic Information System (GIS) approach to generate Ecological Diversity Indices. We used Landscape Ecology Statistics (LecoS v. 3.0.0) 
Table 1. Habitat categories generated by remote sensing and used (1) in the landscape analysis 1. táblázat Távérzékelés során kialakított élőhelykategóriák. Az 1 jelü élőhelytípusok kerültek felhasználásra az elemzésben

\begin{tabular}{|l|c|}
\hline \multicolumn{1}{|c|}{ Habitat type } & $\begin{array}{c}\text { Used for } \\
\text { analysis }\end{array}$ \\
\hline Inland surface waters - standing & 0 \\
\hline Inland surface waters - watercourses & 0 \\
\hline Wetlands with reed, tall herbs & 0 \\
\hline Wetlands with pioneer vegetation & 0 \\
\hline Mires, bogs and fens & 0 \\
\hline Dry grasslands & 1 \\
\hline Mesic grassland, intensively managed & 1 \\
\hline Mesic grassland, medium intensive & 1 \\
\hline Seasonally wet and wet grasslands & 1 \\
\hline Temperate thickets and scrub & 1 \\
\hline Dry heaths & 0 \\
\hline Riverine and fen scrubs & 0 \\
\hline Broadleaved deciduous woodland & 1 \\
\hline Broadleaved evergreen woodland & 0 \\
\hline Coniferous woodland & 1 \\
\hline Lines of trees or hedgerows & 0 \\
\hline Recently felled areas & 0 \\
\hline Arable land and market gardens - intensive & 1 \\
\hline Arable land and market gardens - low intensity & 1 \\
\hline Cultivated areas of gardens and parks & 1 \\
\hline Constructed, industrial and other artificial habitats - with significant green spaces & 1 \\
\hline Constructed, industrial and other artificial habitats - high imperviousness & 1 \\
\hline
\end{tabular}

module in Quantum GIS (QGIS, 3.10.0-A Coruña). LecoS contains several analytical functions for land cover analysis. It can calculate Shannon diversity, evenness, Simpson diversity, perimeter, area and proportion of habitat patches within a polygon. We compared these metrics between countries using Mann-Whitney U-tests.

We applied square-root transformation on the distance variables in order to achieve normality according to Lilliefors tests and QQ-plots. As the data set of Scops Owl observations with regard to distance from paved roads was only partly overlapping with the data set connected to distances from water bodies and settlements, we investigated these variables separately.

We examined the distance from roads using general linear model (GLM). Distance from roads was the dependent variable, country and road were discrete fixed effects, and we investigated the interaction of these too. As tertiary roads and motorways were absent in the Hungarian areas where Scops Owls were detected, we excluded these two categories from the analyses. 
Table 2. Differences of individual habitat variables in Scops Owl observation points between Hungary $(\mathrm{N}=12)$ and Slovenia $(\mathrm{N}=18)$

2. táblázat $A$ magyarországi $(\mathrm{N}=12)$ és szlovéniai $(\mathrm{N}=18)$ füleskuvik megfigyelési pontok körüli élőhelyfoltok fedettségének, arányának és szegélyhosszának összehasonlítása

\begin{tabular}{|c|c|c|c|c|c|}
\hline \multirow{2}{*}{ Variable } & \multicolumn{2}{|c|}{ Mean rank } & \multirow[b]{2}{*}{$\mathbf{U}$} & \multirow[b]{2}{*}{ Z } & \\
\hline & Hungary & Slovenia & & & \\
\hline LP-Dry grasslands & 12.05 & 20.67 & 46.00 & -2.62 & $* *+$ \\
\hline EL-Dry grasslands & 11.72 & 21.17 & 40.00 & -2.88 & $* *+$ \\
\hline MPA-Dry grasslands & 13.56 & 18.42 & 73.00 & -1.48 & \\
\hline LP-Mesic grassland, intensively managed & 19.96 & 12.53 & 54.00 & -2.26 & * \\
\hline EL-Mesic grassland, intensively managed & 20.00 & 12.50 & 54.00 & -2.27 & * \\
\hline MPA-Mesic grassland, intensively managed & 12.11 & 20.58 & 47.00 & 2.58 & $* *+$ \\
\hline LP-Mesic grassland, medium intensive & 15.17 & 16,00 & 102.00 & 0.25 & \\
\hline EL-Mesic grassland, medium intensive & 14.22 & 17.42 & 85.00 & 0.97 & \\
\hline MPA-Mesic grassland, medium intensive & 16,00 & 14.75 & 99.00 & -0.38 & \\
\hline LP-Seasonally wet and wet grasslands & 16.17 & 14.50 & 96.00 & -0.51 & \\
\hline EL-Seasonally wet and wet grasslands & 15.97 & 14.79 & 99.50 & -0.36 & \\
\hline MPA-Seasonally wet and wet grasslands & 17.56 & 12.42 & 71.00 & -1.57 & \\
\hline LP-Temperate thickets and scrub & 16.31 & 14.29 & 93.50 & -0.61 & \\
\hline EL-Temperate thickets and scrub & 16.31 & 14.29 & 93.50 & -0.61 & \\
\hline MPA-Temperate thickets and scrub & 16.05 & 14.67 & 98.00 & -0.42 & \\
\hline LP-Broadleaved deciduous woodland & 15.89 & 14.92 & 101.00 & -0.30 & \\
\hline EL-Broadleaved deciduous woodland & 17.33 & 12.75 & 75.00 & -1.40 & \\
\hline MPA-Broadleaved deciduous woodland & 14.28 & 17.33 & 86.00 & 0.93 & \\
\hline LP-Coniferous woodland & 17.75 & 12.12 & 67.50 & -1.71 & \\
\hline EL-Coniferous woodland & 17.97 & 11.79 & 63.50 & -1.88 & \\
\hline MPA-Coniferous woodland & 17.50 & 12.50 & 72.00 & -1.52 & \\
\hline LP-Arable land and market gardens - intensive & 11.72 & 21.17 & 40.00 & 2.88 & $* *+$ \\
\hline EL-Arable land and market gardens - intensive & 10.50 & 23,00 & 18.00 & 3.81 & $* * * \dagger$ \\
\hline MPA-Arable land and market gardens - intensive & 12.56 & 19.92 & 55.00 & 2.24 & * \\
\hline LP-Arable land and market gardens - low intensity & 10.94 & 22.33 & 26.00 & 3.47 & $* * * \dagger$ \\
\hline EL-Arable land and market gardens - low intensity & 9.83 & 24,00 & 6.00 & 4.32 & $* * * \dagger$ \\
\hline MPA-Arable land and market gardens - low intensity & 12.83 & 19.50 & 60.00 & 2.03 & * \\
\hline LP-Cultivated areas of gardens and parks & 17.75 & 12.13 & 67.50 & -1.71 & \\
\hline EL-Cultivated areas of gardens and parks & 17.17 & 13,00 & 78.00 & -1.27 & \\
\hline MPA-Cultivated areas of gardens and parks & 18.53 & 10.96 & 53.50 & -2.31 & * \\
\hline LP-Constructed habitats - significant green spaces & 15.56 & 15.42 & 107.00 & -0.04 & \\
\hline EL-Constructed habitats - significant green spaces & 14.33 & 17.25 & 87.00 & 0.89 & \\
\hline MPA-Constructed habitats - significant green spaces & 18.33 & 11.25 & 57.00 & -2.16 & * \\
\hline LP-Constructed habitats - high imperviousness & 14.08 & 17.63 & 82.50 & 1.08 & \\
\hline EL-Constructed habitats - high imperviousness & 13.53 & 18.46 & 72.50 & 1.50 & \\
\hline MPA-Constructed habitats - high imperviousness & 16.36 & 14.21 & 92.50 & -0.66 & \\
\hline
\end{tabular}

$\mathrm{LP}$ - landscape proportion, EL - edge length, MPA - mean patch area

${ }^{*} \mathrm{P}<0.05,{ }^{* *} \mathrm{P}<0.01,{ }^{* *} \mathrm{P}<0.001,+\mathrm{P}$ values remained significant after Benjamini-Hochberg FDR correction were marked with bold) 
Table 3. Loadings of the principal components (PCs) performed on habitat variables

3. táblázat A főkomponens (PC) elemzés során az élőhelyjellemzőkre számított származtatott változók

\begin{tabular}{|c|c|c|c|c|c|c|c|c|c|}
\hline Variable & PC1 & PC2 & PC3 & PC4 & PC5 & PC6 & PC7 & PC8 & PC9 \\
\hline LP-Dry grasslands & 0.03 & -0.11 & 0.01 & 0.14 & -0.12 & 0.96 & -0.10 & 0.01 & -0.02 \\
\hline EL-Dry grasslands & 0.11 & -0.18 & 0.04 & 0.37 & -0.17 & 0.85 & -0.11 & 0.00 & -0.06 \\
\hline MPA-Dry grasslands & -0.08 & -0.14 & 0.07 & -0.14 & -0.17 & 0.91 & -0.11 & 0.06 & -0.05 \\
\hline $\begin{array}{l}\text { LP-Mesic grassland, intensively } \\
\text { managed }\end{array}$ & 0.13 & -0.06 & -0.05 & 0.93 & -0.10 & 0.08 & -0.01 & 0.00 & -0.01 \\
\hline $\begin{array}{l}\text { EL-Mesic grassland, intensively } \\
\text { managed }\end{array}$ & 0.07 & -0.10 & -0.02 & 0.94 & -0.13 & 0.12 & 0.01 & 0.05 & -0.01 \\
\hline $\begin{array}{l}\text { MPA-Mesic grassland, intensively } \\
\text { managed }\end{array}$ & 0.20 & -0.17 & 0.00 & 0.85 & -0.25 & 0.05 & -0.07 & 0.02 & -0.10 \\
\hline $\begin{array}{l}\text { LP-Mesic grassland, medium } \\
\text { intensive }\end{array}$ & -0.34 & -0.34 & 0.36 & 0.43 & -0.24 & 0.42 & -0.04 & 0.05 & -0.18 \\
\hline $\begin{array}{l}\text { EL-Mesic grassland, medium } \\
\text { intensive }\end{array}$ & -0.11 & -0.33 & 0.25 & 0.62 & -0.02 & 0.53 & 0.01 & 0.13 & -0.15 \\
\hline $\begin{array}{l}\text { MPA-Mesic grassland, medium } \\
\text { intensive }\end{array}$ & -0.46 & -0.38 & 0.42 & 0.14 & -0.29 & 0.19 & -0.16 & 0.05 & -0.02 \\
\hline $\begin{array}{l}\text { LP-Seasonally wet and wet } \\
\text { grasslands }\end{array}$ & 0.03 & -0.09 & -0.07 & -0.08 & 0.03 & -0.11 & 0.97 & 0.01 & -0.02 \\
\hline $\begin{array}{l}\text { EL-Seasonally wet and wet } \\
\text { grasslands }\end{array}$ & 0.09 & -0.12 & 0.06 & 0.39 & 0.14 & -0.08 & 0.83 & 0.15 & 0.06 \\
\hline $\begin{array}{l}\text { MPA-Seasonally wet and wet } \\
\text { grasslands }\end{array}$ & 0.00 & -0.10 & -0.17 & -0.31 & -0.04 & -0.15 & 0.83 & -0.17 & -0.02 \\
\hline LP-Temperate thickets and scrub & -0.26 & -0.17 & 0.89 & 0.07 & -0.12 & 0.10 & -0.02 & 0.09 & 0.14 \\
\hline EL-Temperate thickets and scrub & -0.30 & -0.21 & 0.82 & 0.15 & -0.13 & 0.20 & 0.02 & 0.17 & 0.04 \\
\hline MPA-Temperate thickets and scrub & -0.14 & -0.17 & 0.48 & -0.03 & -0.18 & 0.01 & 0.02 & 0.16 & 0.78 \\
\hline $\begin{array}{l}\text { LP-Broadleaved deciduous } \\
\text { woodland }\end{array}$ & -0.39 & -0.37 & 0.02 & 0.05 & -0.17 & 0.05 & -0.05 & 0.73 & 0.15 \\
\hline $\begin{array}{l}\text { EL-Broadleaved deciduous } \\
\text { woodland }\end{array}$ & -0.48 & -0.46 & 0.28 & 0.10 & -0.15 & 0.02 & -0.05 & 0.61 & -0.16 \\
\hline $\begin{array}{l}\text { MPA-Broadleaved deciduous } \\
\text { woodland }\end{array}$ & -0.11 & -0.20 & 0.02 & 0.07 & -0.15 & 0.03 & 0.01 & 0.55 & 0.71 \\
\hline LP-Coniferous woodland & -0.45 & -0.29 & 0.54 & -0.18 & -0.34 & -0.10 & -0.23 & 0.22 & -0.22 \\
\hline EL-Coniferous woodland & -0.48 & -0.33 & 0.49 & -0.19 & -0.36 & -0.10 & -0.23 & 0.28 & -0.22 \\
\hline MPA-Coniferous woodland & -0.18 & -0.17 & 0.84 & -0.17 & -0.17 & -0.07 & -0.15 & 0.01 & 0.06 \\
\hline $\begin{array}{l}\text { LP-Arable land and market gardens } \\
\text { - intensive }\end{array}$ & 0.74 & -0.18 & -0.24 & -0.15 & -0.25 & -0.19 & -0.06 & -0.24 & 0.38 \\
\hline $\begin{array}{l}\text { EL-Arable land and market gardens } \\
\text { - intensive }\end{array}$ & 0.90 & -0.11 & -0.24 & -0.01 & -0.02 & -0.09 & -0.07 & -0.21 & -0.02 \\
\hline $\begin{array}{l}\text { MPA-Arable land and market } \\
\text { gardens - intensive }\end{array}$ & 0.17 & -0.14 & -0.08 & -0.18 & -0.11 & -0.19 & -0.01 & -0.07 & 0.90 \\
\hline $\begin{array}{l}\text { LP-Arable land and market gardens } \\
\text { - low intensity }\end{array}$ & 0.90 & -0.06 & -0.14 & 0.20 & 0.14 & 0.08 & 0.18 & 0.17 & -0.10 \\
\hline $\begin{array}{l}\text { EL-Arable land and market gardens } \\
\text { - low intensity }\end{array}$ & 0.92 & -0.08 & -0.15 & 0.28 & -0.10 & 0.12 & -0.01 & -0.05 & -0.06 \\
\hline $\begin{array}{l}\text { MPA-Arable land and market } \\
\text { gardens - low intensity }\end{array}$ & 0.39 & -0.08 & -0.01 & -0.03 & 0.62 & -0.02 & 0.37 & 0.49 & 0.05 \\
\hline
\end{tabular}




\begin{tabular}{|l|c|c|c|c|c|c|c|c|c|}
\hline \multicolumn{1}{|c|}{ Variable } & PC1 & PC2 & PC3 & PC4 & PC5 & PC6 & PC7 & PC8 & PC9 \\
\hline $\begin{array}{l}\text { LP-Cultivated areas of gardens and } \\
\text { parks }\end{array}$ & -0.13 & 0.01 & -0.19 & -0.21 & $\mathbf{0 . 8 9}$ & -0.21 & 0.00 & -0.19 & -0.13 \\
\hline $\begin{array}{l}\text { EL-Cultivated areas of gardens and } \\
\text { parks }\end{array}$ & -0.13 & -0.01 & -0.23 & -0.20 & $\mathbf{0 . 8 1}$ & -0.21 & 0.13 & -0.31 & -0.02 \\
\hline $\begin{array}{l}\text { MPA-Cultivated areas of gardens } \\
\text { and parks }\end{array}$ & -0.04 & -0.02 & -0.11 & -0.18 & $\mathbf{0 . 9 2}$ & -0.17 & -0.05 & 0.08 & -0.10 \\
\hline $\begin{array}{l}\text { LP-Constructed habitats - } \\
\text { significant green spaces }\end{array}$ & -0.23 & $\mathbf{0 . 7 6}$ & -0.28 & -0.15 & 0.17 & -0.22 & -0.02 & -0.37 & -0.16 \\
\hline $\begin{array}{l}\text { EL-Constructed habitats - } \\
\text { significant green spaces }\end{array}$ & 0.06 & 0.51 & -0.36 & -0.08 & 0.15 & -0.13 & -0.09 & $-\mathbf{0 . 6 1}$ & -0.29 \\
\hline $\begin{array}{l}\text { MPA-Constructed habitats - } \\
\text { significant green spaces }\end{array}$ & -0.18 & $\mathbf{0 . 8 9}$ & -0.06 & -0.08 & -0.14 & -0.09 & -0.06 & 0.09 & -0.02 \\
\hline $\begin{array}{l}\text { LP-Constructed habitats - high } \\
\text { imperviousness }\end{array}$ & -0.02 & $\mathbf{0 . 9 2}$ & -0.15 & -0.14 & 0.01 & -0.14 & -0.11 & -0.19 & -0.14 \\
\hline $\begin{array}{l}\text { EL-Constructed habitats - high } \\
\text { imperviousness }\end{array}$ & 0.02 & $\mathbf{0 . 9 3}$ & -0.16 & -0.13 & -0.04 & -0.14 & -0.12 & -0.16 & -0.13 \\
\hline $\begin{array}{l}\text { MPA-Constructed habitats - high } \\
\text { imperviousness }\end{array}$ & -0.07 & 0.35 & 0.26 & 0.06 & 0.10 & -0.01 & 0.29 & -0.41 & -0.27 \\
\hline Eigenvalue & 4.78 & 4.78 & 4.06 & 4.02 & 3.68 & 3.43 & 2.83 & 2.74 & 2.66 \\
\hline Explained variance (\%) & 13.29 & 13.26 & 11.22 & 11.16 & 10.22 & 9.53 & 7.87 & 7.60 & 7.38 \\
\hline Cumulative variance (\%) & 13.29 & 26.55 & 37.77 & 48.93 & 59.15 & 68.68 & 76.55 & 84.15 & 91.53 \\
\hline
\end{tabular}

Loading values which exceeded the lower threshold of 0.60 were marked with bold.

Table 4. Differences of habitat principal components (PCs) in Scops Owl observation points between Hungary $(\mathrm{N}=12)$ and Slovenia $(\mathrm{N}=18)$

4. táblázat $A z$ élőhelyre vonatkozó főkomponensek (PC) közötti különbségek a magyar $(\mathrm{N}=12)$ és a szlovén $(\mathrm{N}=18)$ területeken található füleskuvik észlelési pontok körül

\begin{tabular}{|l|c|c|c|c|c|}
\hline & \multicolumn{2}{|c|}{ Mean rank } & & & \\
\hline Variable & Hungary & Slovenia & $\mathbf{U}$ & $\mathbf{Z}$ & \\
\hline PC1 & 10.50 & 23.00 & 18.00 & 3.81 & $* * *$ \\
\hline PC2 & 14.17 & 17.50 & 84.00 & 1.02 & \\
\hline PC3 & 15.78 & 15.08 & 103.00 & -0.21 & \\
\hline PC4 & 14.44 & 17.08 & 89.00 & 0.80 & \\
\hline PC5 & 16.61 & 13.83 & 88.00 & -0.85 & \\
\hline PC6 & 13.94 & 17.83 & 80.00 & 1.19 & \\
\hline PC7 & 16.22 & 14.42 & 95.00 & -0.55 & \\
\hline PC8 & 15.17 & 16,00 & 102.00 & 0.25 & \\
\hline PC9 & 14,00 & 17.75 & 81.00 & 1.14 & \\
\hline
\end{tabular}

Table 5. Differences of habitat diversity indices in Scops Owl observation points between Hungary $(\mathrm{N}=12)$ and Slovenia $(\mathrm{N}=18)$

5. táblázat Az ökológiai diverzitás mutatószámaiban mérhető különbségek a magyarországi $(\mathrm{N}=12)$ és a szlovéniai $(\mathrm{N}=18)$ területeken található füleskuvik észlelési pontok körül

\begin{tabular}{|l|c|c|c|c|}
\hline \multirow{2}{*}{ Variable } & \multicolumn{2}{|c|}{ Mean rank } & & \\
\cline { 2 - 5 } & Hungary & Slovenia & $\mathbf{U}$ & $\mathbf{Z}$ \\
\hline $\begin{array}{l}\text { Shannon } \\
\text { diversity }\end{array}$ & 13.72 & 18.17 & 76.00 & -1.35 \\
\hline Evenness & 13.33 & 18.75 & 69.00 & -1.65 \\
\hline $\begin{array}{l}\text { Simpson } \\
\text { diversity }\end{array}$ & 13.22 & 18.92 & 67.00 & -1.74 \\
\hline
\end{tabular}


Using GLMs, we also explored if there is any difference in distances (from roads, settlements and water bodies) between real field observations and randomly selected points. In each GLM, one of the four distance variables was the dependent variable, country and observation type (real $v s$. randomized) were used as discrete fixed effects, and the initial model included the interaction of these factors.

Random spatial points were generated by QGIS (vector/research tools/random points inside polygons) within those $2.5 \times 2.5 \mathrm{~km}$ UTM quadrates where Scops Owls were absent.

For GLMs, we applied backward stepwise model simplification (Hegyi \& Laczi 2015). We performed the statistical analyses in Statistica 7.0 (Statsoft, Inc.).

\section{Results}

\section{Environmental variables}

We detected significant differences between countries in seven habitat variables: proportion and edge length of dry grasslands were lower in Hungary, as well as the mean patch area of intensively managed mesic grasslands, proportions and edge lengths of intensive and low intensity arable land and market gardens (Table 2). The PCA resulted in nine PCs with eigenvalue larger than one (Table 3). Higher value of a given PC means larger values in the original variables with positive loadings, and lower values in the original variables with negative loadings. According to this, e.g. higher PC1 represents larger proportions and edge lengths of intensive and low intensity arable land and market gardens. Between countries, only PC1 differed significantly, i.e. it was higher in Slovenia (Table 4), which is partly in concordance with the previous results. Ecological (landscape) diversity indices did not differ between the two countries (Table 5).

\section{Distances from roads}

Analyses revealed that Scops Owls occupied habitats slightly farther from roads in Hungary than in Slovenia $\left(\mathrm{F}_{1,48}=4.54, \mathrm{P}=0.038\right.$, mean $\pm \mathrm{SD}: 46.74 \pm 7.39$ in Hungary, $44.28 \pm$ 4.33 in Slovenia), independently of road types (primary and secondary). However, road type also had a significant effect $\left(\mathrm{F}_{1,48}=176.74, \mathrm{P}<0.0001\right)$ because Scops Owls were observed further from primary roads than from secondary roads (Figure 2), independently of country. The 'road type $\mathrm{x}$ country' interaction was not significant $\left(\mathrm{F}_{1,47}=2.15, \mathrm{P}=0.15\right)$.

\section{Distance from settlements and water bodies}

Comparing real and randomized data, GLMs revealed that distances were smaller in the case of real observations from primary roads (mean \pm SE, real: $66.14 \pm 4.48$, random: 90.84 \pm 5.15 ), secondary roads (real: $17.73 \pm 2.08$, random: $31.02 \pm 2.70$ ) and settlements (real: $34.62 \pm 3.52$, random: $50.67 \pm 4.04$ ) irrespective of country, but there was no difference in distances from water bodies (real: $16.20 \pm 1.40$, random: 19.10 \pm 1.58 ) (Table 6). 


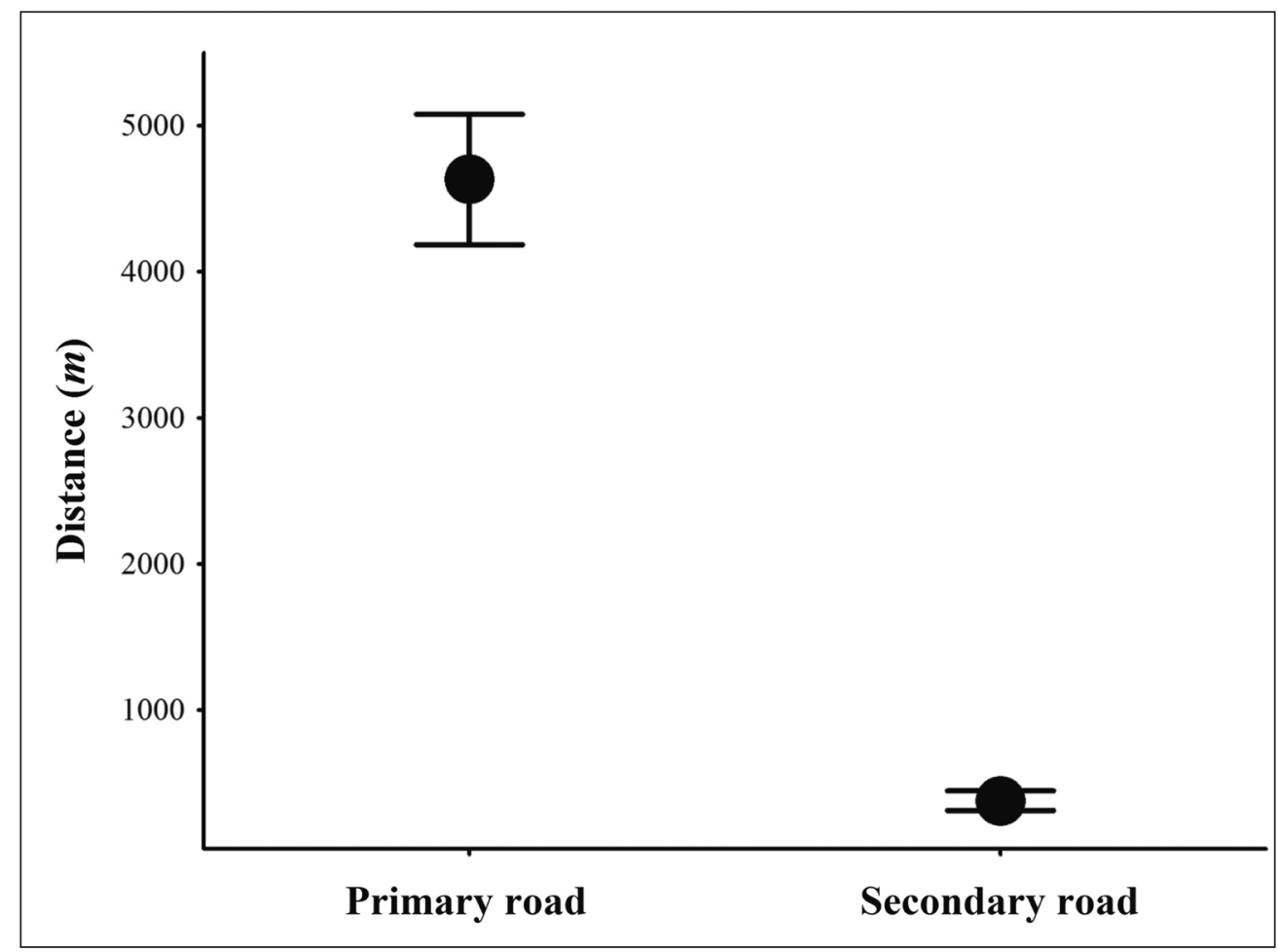

Figure 2. Distance of Scops Owl observations from different road types in Hungary and Slovenia. For illustration, we presented the raw distance data (mean \pm SE)

2.ábra Különböző úttípusoktól mért távolságok. A szemléltetéshez a valós távolsági adatokat (átlag \pm SE) ábrázoltuk

\section{Discussion}

Several studies attempted to explain the population decline of the Scops Owl in Europe (Sergio et al. 2009, Treggiari et al. 2013, Denac et al. 2019). Landscape transformation and the change in agricultural use undoubtedly lead the list of human induced factors, however, revealing these effects still remains a real challenge in Central Europe, outside the core Mediterranean range (Ivajnšič et al. 2020).

Sergio et al. (2009) concludes that Scops Owl abundance in the Central-Eastern Italian pre-Alps is determined by two factors. Scops Owl territories differed from randomly generated locations in two respects: (1) extensive agriculture (length of hedgerows and availability of individual trees) and (2) proximity to the Tawny Owl Strix aluco territories, its main predator.

It has previously been tested and proved that the abundance of the Scops Owl is associated with a mosaic of land-use categories (Denac et al. 2019). Although there are well visible differences in landscape structure between GNP and Vas County, we did not find evidence that these differences were associated with the difference in the Scops Owl carrying capacity of the two regions. The agricultural conditions in Slovenia allowed the preservation of 
the patchwork-like landscape with large number of small $(<2 \mathrm{ha})$ plots managed by different farmers. This landscape structure is able to maintain high versatility of various cultivations, large network of unmanaged hedges and verges and consequently, rich biodiversity. By contrast, in Hungary more than $95 \%$ of agricultural land had become the property of collective farms between 1945 and 1961 (Swain 1985) that ultimately resulted in a transformed landscape structure dominated by large monocultures across the whole of Hungary. Vas County is regarded as one of the regions in Hungary that managed to save a remarkable proportion of the mosaic landscape, which may explain the lack of significant difference in the ecological diversity indices between GNP and Vas County in our study.

The average size of habitat plots and the total length of edges per se are certainly important features, but the actual composition of differently farmed plots in the landscape might tell even more about the suitability of a habitat for Scops Owls. We found that both proportion and edge length of dry grasslands and intensively managed mesic grasslands were lower in Hungary. Also, market gardens were present in a larger proportion in GNP. These landscape features all indicate that in GNP the complex cultivation is still pronounced, and less intensive cultivation modes, like rural market gardens and grasslands play a key role in Sops Owl habitat selection.

The dire decline of Scops Owl population over the past 25 years in GNP (Štumberger 2000, Denac et al. 2019) warns conservationists and policy makers that adverse landscape transformation reaches even the last strongholds of our sensitive iconic species. By modelling Scops Owl breeding suitability in GNP, Ivajnšič et al. (2020) outlined a predictable significant population decrease (by 33\%) in GNP in the next 50 years. They discuss in detail that EU Common Agricultural Policy (CAP) is poorly adapted to the needs of Central and Eastern European low-intensity farming traditions, especially with regards to the high biodiversity grasslands. At the European level, an adapted CAP and specifically tailored subsidy schemes can support farmers to maintain large landscape complexity. However, to our opinion such interventions are only able to expand the agony of the post-rural countryside in Central Europe. Current food production policy, expensive agricultural technology and global trade agreements all mean impediments to a self-sustained small-scale agricultural practice that could restore biodiversity focused farming and local communities long-term.

Before such structural economic and societal changes take place, cost and time effective conservation interventions are needed to ensure the viability of endangered bird populations. In agreement with the conclusions of Sergio et al. (2009), who pointed out the importance of the individual trees and network of hedges, we recommend the establishment of a network of Veteran Tree Reserves (VTRs) in Vas County. The Hungarian landscape, unlike that of some other European countries as England, lack old trees. Historical, cultural and legislative factors played a role over the past two centuries in that most of the ancient tree specimens along with the network of hedges and unmanaged verges disappeared from the rural countryside. Hedges and solitary trees hold value both for biodiversity and landscape if their lifespan overarch centuries. This longevity was ensured in the United Kingdom since the early medieval era, as hedges and planted trees displayed field boundaries and the preservation of these landscape features became an organic part of the culture, up to the $1950 \mathrm{~s}$, when post-war industrial agriculture eliminated a great deal of these wildlife 
corridors (Barnes \& Williamson 2006). Newly established VTRs would increase connectivity between cross-border subpopulations and between individual breeding pairs, and they would provide suitable calling, breeding and foraging habitats for Scops Owls.

The network of VTRs can be improved even if the coexistence of Tawny Owl and Scops Owl have not been investigated in Hungary yet. The Tawny Owl is a common nocturnal predator in W Hungary, whereas its presence is less pronounced in C Hungary, where the most stable Scops Owl populations exist (MME 2020). However, further investigations are needed to clarify how certain locations in Vas County serve as traditional Scops Owl breeding sites for decades, where Tawny Owls are permanently present as well.

A possible explanation can be the protection provided by the urban environment. Scops Owl observations in this study appeared to be closer to settlements than the ones randomly generated in the landscape. It can be assumed that Tawny Owls hunt less frequently in settlements. Streetlight in urban environment also can attract Scops Owls closer to settlements, although there was no correlation between spatial breeding distribution and light pollution patterns in GNP (Ivajnšič et al. 2020). As Sergio et al. (2007) pointed out, intraguild predation among owl species is density dependent. There might be a threshold of abundance, beyond which the effect of Tawny Owl predation risk on Scops Owl occurrence becomes significant.

Moreover, Scops Owls were observed farther from primary roads than from secondary roads. This is in accordance with other studies showing that corticosterone level was higher in owlets hatched closer to roads (Expósito-Granados et al. 2019). Šušmelj (2011) also concluded that larger distance from highways increased the likelihood of Scops Owl settlement in the Slovenian Karst.

Conservation effort should be prioritised in an era, where ecological and climate crisis sweeps thousands of species away. Conserving range edge populations is important as these populations are the best subjects for fast adaptation and speciation. However, investing disproportional capacities in the reinforcement of sink populations when the source ones are under dire threat, needs to be reconsidered. The deployment of cost-effective and sustainable conservation measures, like the introduction of VTRs network, is an obvious step to take, but ultimately the solutions must urge the halt of further large-scale landscape transformations at the policy level.

\section{Acknowledgements}

The fieldwork was funded by the INTERREG Danube Transnational Programme DTP2007-2.3. We thank MME - BirdLife Hungary, the team of Birding.hu, Örség National Park staff and several field ornithologists for providing Scops Owl data from Hungary. Katerine Denac (DOPPS, BirdLife - Slovenia) offered kindly data and information of the Goričko Scops Owl population. 


\section{References}

Barnes, W. \& Williamson, T. 2006. Hedgerow History: Ecology, History and Landscape Character. - Windgather Press, Cheshire

Barthos, Gy. 1957. Breeding Scops Owl in South-Western Hungary. - Aquila 63-64: 288-289., 344-345. (in Hungarian with English Summary)

Benjamini, Y. \& Hochberg, Y. 1995. Controlling the false discovery rate: a practical and powerful approach to multiple testing. - Journal of the Royal Statistical Society B 57(1): 289-300.

BirdLife International 2020. Species factsheet: Otus scops. - http://www.birdlife.org

Davies, C. E., Moss, D. \& Hill, M. O. 2004. EUNIS Habitat Classification Revised 2004. Report to the European Topic Centre on Nature Conservation, European Environment Agency, October 2004.

Denac, K., Kmecl, P. \& Koce, U. 2019. Habitat use of Eurasian Scops Owls Otus scops in an agricultural mosaic landscape. - Ardea 107(2): 119-129. DOI: 10.5253/arde.v107i2.a1

Expósito-Granados, M., Parejo, D., Chastel, O. \& Avilés, J. M. 2019. Physiological stress and behavioural responses of European Rollers and Eurasian Scops Owls to human disturbance differ in farming habitats in the south of Spain. - Bird Conservation International, Cambridge University Press, pp. 1-16. DOI: 10.1017/ S0959270919000388

Gill, F. \& Donsker, D. 2019. IOC World Bird List (v 9.2). - DOI: 10.14344/IOC.ML.9.2.

Gaston, K. 2009. Geographic range limits: Achieving synthesis. - Proceedings of the Royal Society B: Biological Sciences 276: 1395-1406. DOI: 10.1098/rspb.2008.1480

Hadarics, T. \& Zalai, T. 2008. Nomenclator Avium Hungariae Magyarország Madarainak Névjegyzéke - An annonated list of the birds of Hungary. - MME Birdlife Hungary, Budapest (in Hungarian with English Summary)

Hegyi, G. \& Laczi, M. 2015. Using full models, stepwise regression and model selection in ecological data sets: Monte Carlo simulations. - Annales Zoologici Fennici 52: 145-159. DOI: 10.5735/086.052.0502

Ivajnšič, D., Denac, D., Denac, K., Pipenbaher, N. \& Kaligarič, M. 2020. The Scops Owl (Otus scops) under human-induced environmental change pressure. - Land Use Policy 99: 104853. DOI: 10.1016/j.landusepol.2020.104853

MME 2020 - Magyar Madártani és Természetvédelmi Egyesület. Magyarország madarai. - BirdLife Hungary, The Birds of Hungary on-line database. - https://www.mme.hu/magyarorszagmadarai/madaradatbazis (in Hungarian)

Martínez, J. A., Zuberogoitia, I., Martínez, J. E., Zabala, J. \& Calvo, F. 2007. Patterns of territory settlement by Eurasian Scops-owls (Otus scops) in altered semi-arid landscapes. - Journal of Arid Environments 69(3): 400-409. DOI: 10.1016/j.jaridenv.2006.10.011

Randik, A. 1959. The Distribution of the Scops Owl in the Carpathian Basin. - Aquila 66: 99-106., 104-106. (in Hungarian with English Summary)

Sergio, F., Marchesi, P., Pedrini, P. \& Penteriani, V. 2007. Coexistence of a generalist owl with its intraguild predator: distance-sensitive or habitat-mediated avoidance? - Animal Behaviour 74: 1607-1616. DOI: 10.1016/j.anbehav.2006.10.022

Sergio, F., Marchesi, L. \& Pedrini, P. 2009. Conservation of Scops Owl Otus scops in the Alps: relationships with grassland management, predation risk and wider biodiversity. - Ibis 151(1): 40-50. DOI: 10.1111/j.1474919X.2008.00865.x.

Štumberger, B. 2000. Veliki skovik Otus scops na Goričkem [Eurasian Scops Owl Otus scops at Goričko (NE Slovenia)]. - Acrocephalus 21: 23-29. (in Slovenian)

Šušmelj, T. 2011. The impact of environmental factors on distribution of Scops Owl Otus scops in the wider area of Kras (SW Slovenia). - Acrocephalus 32(148-149): 11-28.

Swain, N. 1985. Collective Farms Which Work? - Cambridge University Press, Cambridge

Treggiari, A. A., Gagliardone, M., Pellegrino, I. \& Cucco, M. 2013. Habitat selection in a changing environment: the relationship between habitat alteration and Scops Owl (Aves: strigidae) territory occupancy. - Italian Journal of Zoology 80(4): 574-585. DOI: 10.1080/11250003.2013.853843 